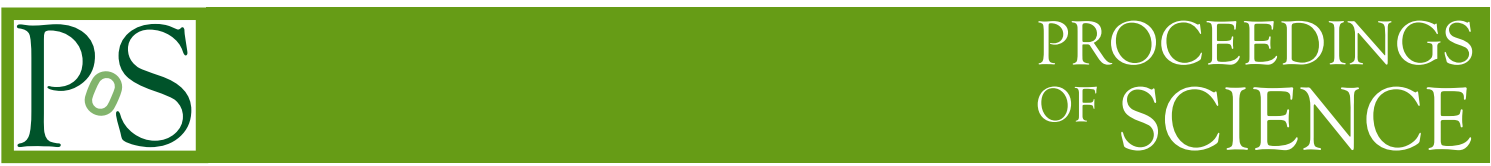

\title{
Tracking and flavour tagging performance at ATLAS
}

\section{Carlo Varni, on behalf of the ATLAS collaboration ${ }^{a, *}$}

${ }^{a}$ INFN and Università degli Studi di Genova

E-mail: carlo.varni@cern.ch

The identification of jets originating from heavy-flavour quarks $(b, c)$ is central to the LHC physics program. High-performance flavour tagging is necessary both in precise Standard Model measurements and in searches for new physics. To achieve this, distinct characteristics of heavyflavour decays are exploited, such as the presence of secondary vertices and displaced particles. This requires an accurate picture of the charged particle activity within jets, which is obtained performing efficient and precise track reconstruction in the ATLAS Inner Detector. After providing details on the role of track reconstruction in $b$-tagging, we present the latest heavy-flavour jet tagging algorithms developed by the ATLAS collaboration. In addition we report, for both track reconstruction and flavour tagging tasks, recent performance results as expected in simulation and as measured in collision data.

40th International Conference on High Energy physics - ICHEP2020

July 28 - August 6, 2020

Prague, Czech Republic (virtual meeting)

${ }^{*}$ Speaker 


\section{Introduction}

Flavour tagging is a tool that aims at correctly identifying and separating jets stemming from light quarks or gluons (light-flavour jets), and heavy quarks ( $c$ - or $b$-jets). The differences between these jets reflect the properties of heavy-flavoured hadrons: a long lifetime, which leads to a displaced (or secondary) vertex; and large impact parameters and mass. As a consequence, an efficient and reliable track reconstruction and a precise measurement of vertices' position are pivotal elements for flavour tagging.

In this contribution, a quick description of tracking performance within jets is presented, as well as a description of the main flavour tagging algorithms developed by the ATLAS [1] Collaboration. Also, flavour tagging strategies for high transverse momentum $\left(p_{T}\right)$ jet will be discussed.

\section{Tracking within jets}

The Inner Detector (ID), which is responsible for reconstructing particle trajectories, combines information from three subdetectors that use different tracking technologies: pixel sensors, silicon microstrip sensors and straw tubes. The first step of the ATLAS track reconstruction [2] consists of grouping energy deposits from the interaction of the charged particles with the detector layers into clusters of energies from raw measurements. However, the majority of charged particles within highly energetic hadronic jets are concentrated in the jet core, as shown in the top-left plot of Figure 1. In such a dense environment the average charged particle separation is comparable to the granularity of individual sensor elements of the ID resulting in overlaps of charge deposits from multiple particles. This leads to the occurrence of so-called merged clusters: energy clusters created by charge deposits from multiple particles. Merged clusters affect the track reconstruction algorithm performance and limit tracking efficiency. An efficient identification of merged clusters is pivotal for an efficient charged particle reconstruction.

After cluster creation, a staged pattern recognition approach is used: a loose track candidate search provides combinatorial track candidates to a stringent ambiguity-solver, which resolves any ambiguity with respect to duplicated hits or hits wrongly attributed to tracks. The ambiguity-solver uses an artificial Neural Network (NN) trained to identify merged clusters in order to maximise tracking efficiency. Finally, a high-resolution fit is performed using all available information.

The plots in Figure 1 illustrate the loss of track reconstruction efficiency due to the wrong identification of merged clusters. The track reconstruction efficiency varies according to the angular distance between the charged particle and the jet axis. The closer the particles to the jet core, the more significant the drop of efficiency is due to the high particle density. This effect is exacerbated at high transverse momentum, where charged particles are more collimated. A decrease in efficiency with production radius (defined as the radial distance from the beam axis) is also observed. This is due to two different effects. Firstly, at large production radius the charged particles are created beyond the first active layers of the Inner Detector, thus creating fewer clusters. Secondly, charged particles are created very close to the next active layer of the ID, with high- $p_{T}$ and collimated. Thus, leading to the creation of more merged clusters. The same effect is observed at all pseudorapidity values $(\eta)$, but the loss of efficiency is more dramatic at high $\eta$. 

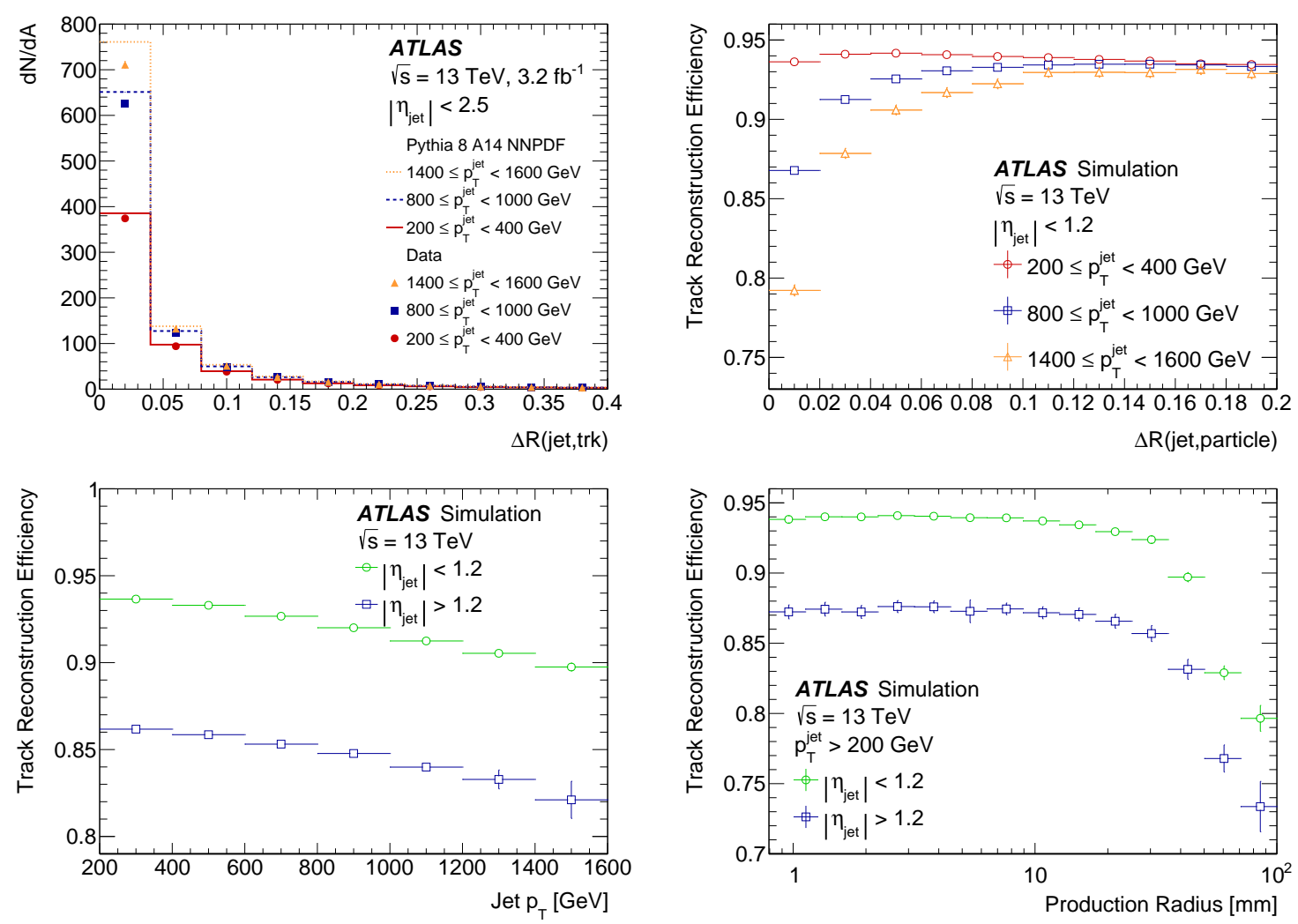

Figure 1: The average number of primary tracks as a function of the angular distance from the jet axis (top-left plot) and the track reconstruction efficiency as a function of the angular distance from the jet axis (top-right plot), of $p_{T}$ (bottom-left plot) and production radius (bottom-right plot) [2].

\section{Flavour Tagging Algorithms}

The differences between $b$-, $c$ - or light-flavour jets is reflected in several physical quantities. Specialised algorithms have been developed to analyse these discriminating quantities [3]: IP2D and IP3D, that analyse track $d_{0}$ and $z_{0}$ significances and their correlations; SV1, that reconstructs discriminating variables related to the presence of a single and inclusive secondary vertex; and JetFitter, that reconstructs the complete $b$-hadron decay chain. The outputs from these complementary algorithms are then combined in high-level taggers, resulting in more versatile and powerful tools. The MV2 algorithm - a Boosted Decision Tree (BDT) - has been widely used during Run 2, but recently the DL1 algorithm - based on a NN architecture - has been introduced. Also in recent years, a new low-level impact parameter-based tagger has been implemented, which exploits correlations between the tracks using a Recurrent Neural Network (RNN) approach: RNNIP [4]. High-level tagger variants, which also exploit the RNNIP output, have been made available and DL1 $r$ is the currently recommended flavour tagging algorithm in ATLAS.

The use of a NN is preferable since it provides more flexibility with respect to a Boosted Decision Tree. A BDT provides a single output discriminant and the training and testing of the algorithm is obtained for a specific signal and background composition. A different background composition would require a different training of the algorithm. This was the case for the different 
specializations of the MV2 algorithm for different $c$-quark background contaminations. Conversely, a NN provides three output nodes, corresponding to the probability that the jet is stemming from a light-, $c$ - or $b$-quark: $p_{u}, p_{c}$ and $p_{b}$ respectively. The final discriminating variable DL1r, defined by the Eq. 1 , is given by a log likelihood ratio (LLR) in which a free parameter - the charm fraction $\left(c_{f}\right)$ - can be tuned in order to find the optimal trade-off between rejection of charm and light-flavour jets. This can be done without the need to produce a new ad-hoc training, since the NN treats all flavours equally.

$$
\operatorname{DL} 1 \mathrm{r}=\log \left(\frac{p_{b}}{p_{c} c_{f}+p_{u}\left(1-c_{f}\right)}\right)
$$

This can also be extended to target the separation of $c$-jets from light-flavour and $b$-jets - i.e. charm-tagging - by only defining a different signal and background in the LLR, see Eq.2. The background composition is defined by a different free parameter: the bottom fraction $\left(b_{f}\right)$.

$$
\mathrm{DL} 1 \mathrm{r}_{\mathrm{c}}=\log \left(\frac{p_{c}}{p_{b} b_{f}+p_{u}\left(1-b_{f}\right)}\right)
$$

A new algorithm may replace RNNIP in the future: the Deep Impact Parameter Sets (DIPS) [5]. This algorithm solves the same task as RNNIP, but does not require any specific order of the input elements, which is better physically motivated since $b$-hadron decay products do not exhibit any intrinsic sequential ordering.

\section{Flavour Tagging Performance}

The performance of the $b$-tagging algorithms with respect to the true flavour of jets is expressed in terms of $l i g h t$-flavour jet and $c$-jet rejection as a function of $b$-jet efficiency. Figure 2 illustrates the performance of the main high-level algorithms, both for light-flavour and $c$-jet rejection. In both cases DL1r outperforms the other taggers, demonstrating that the introduction of the RNNIP algorithm helped improving the flavour tagging performance. Background rejection, at a given $b$-jet efficiency, decreases at high jet $p_{T}$. The training and testing phases of the flavour tagging algorithms are performed on a mixture of $t \bar{t}$ and $Z^{\prime}$ (mass of $1000 \mathrm{GeV}$ ) simulated events [6]. Since this ensures a training sample with high statistics in a broad $p_{T}$ spectrum, it mitigates the decrease of performance at high- $p_{T}$, albeit still present.

The flavour tagging performance must be calibrated before being used in any physics analysis. MC-to-data weights (Scale factors) - measured in both data and simulated $t \bar{t}$ events - are applied to Monte Carlo simulations to correct for any mismodelling of flavour tagging performance. The calibration on $b$-jets is based on a likelihood-based method, as described in Refs. [7, 8].

\section{Boosted regimes}

At high momenta, the decay products of heavy resonances are highly collimated and are merged into large radius (large-R) jets. Focusing on the decay to bottom quark pairs, the ability to reconstruct Large-R jets and the decay products (sub-jets) is therefore crucial for improving the sensitivity of dedicated searches. This is the case for a boosted Higgs boson decaying to pairs of bottom quarks, which is of fundamental importance for the ATLAS physics program. 

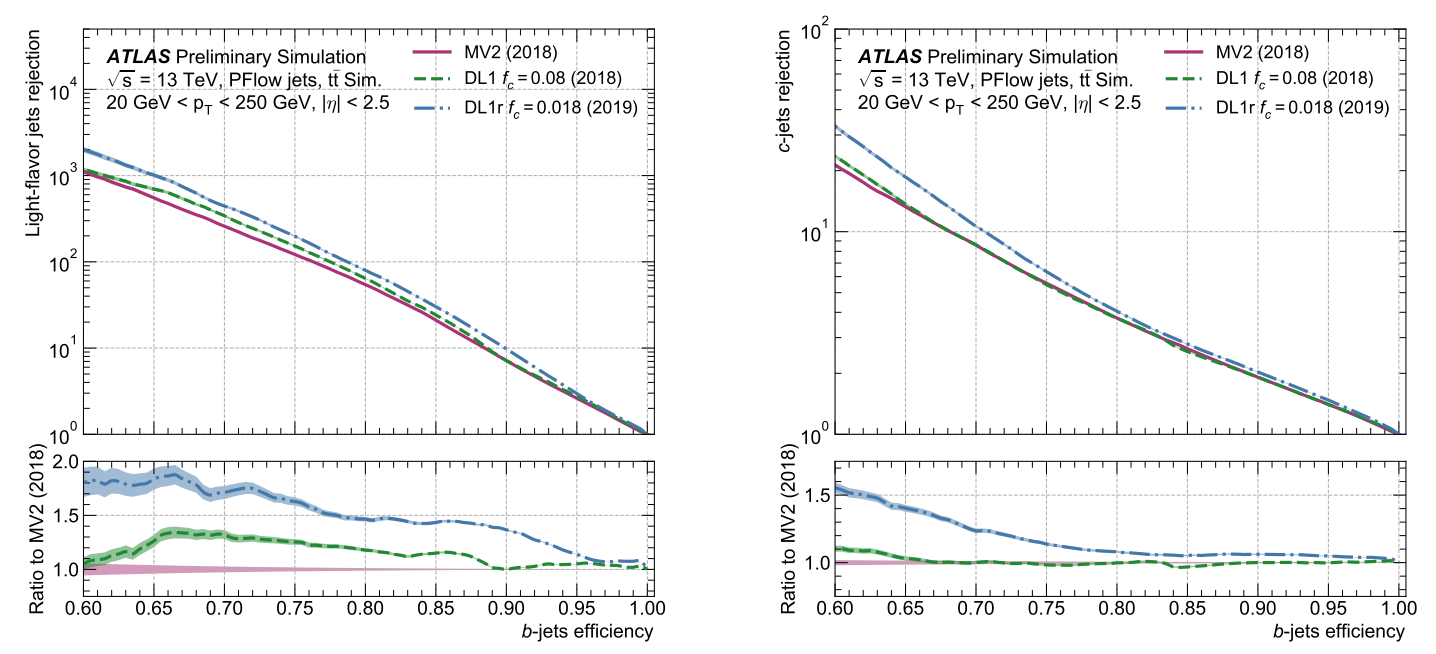

Figure 2: Comparison of ROC (Receiver Operating Characteristic) curves for light-jet (left plot) and $c$-jet rejection (right plot) as a function of $b$-jet efficiency for the MV2, DL1, and DL1r algorithms [9].
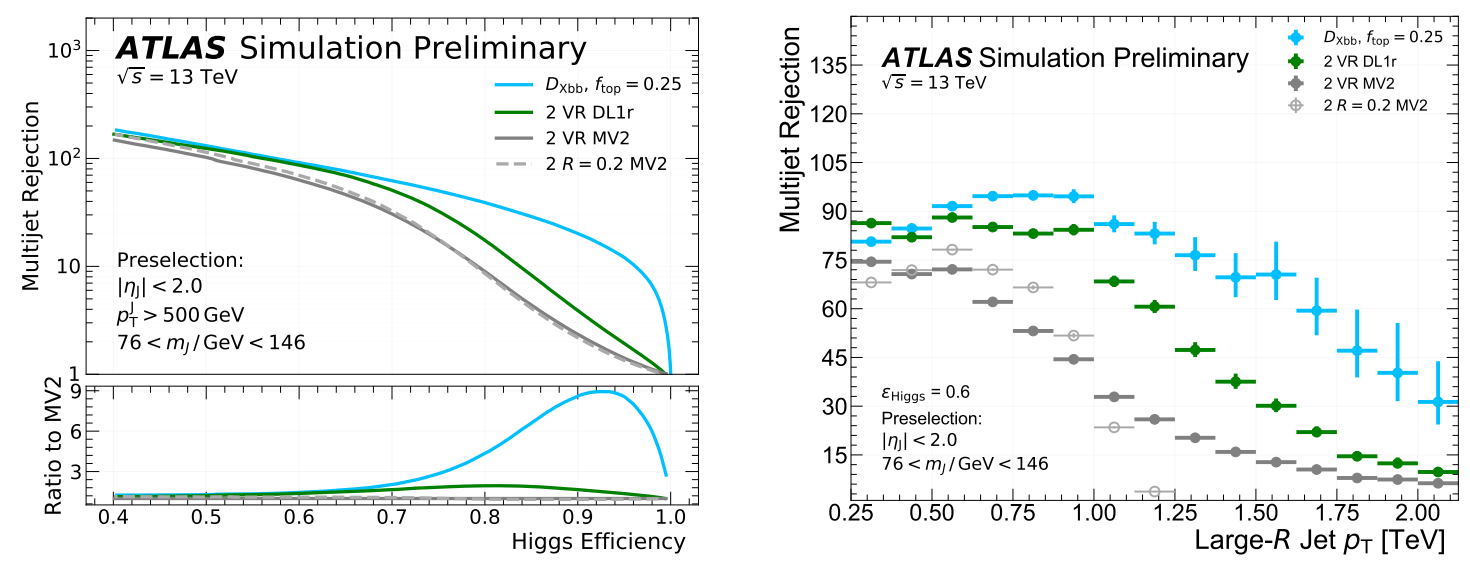

Figure 3: Multi-jet rejection as a function of the $H \rightarrow b \bar{b}$ tagging efficiency (left plot) and $p_{T}$ for large-R jet (right plot). Performance of the algorithm is compared to DL1r and to two variants of MV2: with variable-radius jets and fixed-radius jets [11].

The baseline sub-jet reconstruction algorithm in ATLAS utilises a fixed radius parameter $(R=$ $0.2)$ and uses tracks as inputs. The fixed-radius approach is adequate as long as the hadronisation products of the two $b$-quarks from the Higgs boson decay do not overlap. When that happens, the efficiency of the sub-jet reconstruction algorithm severely drops and it is not able anymore to resolve the large-R jet substructures [10]. An alternative approach, which uses a variable radius (VR) jet algorithm, compensates for this inefficiency. This algorithm parametrizes the radius $R=\rho / p_{T}$ as a function of the jet $p_{T}$, where $\rho$ is a constant, and imposes lower and upper cut-offs on the jet size.

This can be extended also to $b$-tagging with a new NN-based tagging algorithm, optimized for the $H \rightarrow b \bar{b}$ topology, that combines the flavour information of up to three sub-jets within the large- $\mathrm{R}$ jet [11]. This algorithm takes advantage of the tagging performance for individual 
sub-jets and their discriminant correlations, as well as the large- $\mathrm{R}$ jet kinematics. The resulting discriminating variable $\left(D_{X b b}\right)$ provides the ability to reject boosted top quark jets and jets arising from multi-jet processes. The trade-off between the rejection of these two backgrounds is adjusted via a free parameter, $f_{\text {top }}$, that defines the background composition in the LLR definition. The performance of such an algorithm are shown in Figure 3.

\section{Conclusions}

A description of tracking and flavour tagging in ATLAS has been presented. Tracking constitutes a pivotal element in flavour tagging and an efficient track reconstruction within jets is of fundamental importance, despite its complexity due to the dense environment. The main flavour tagging algorithms adopted by ATLAS have been introduced as well as their performance, explaining the advantages in moving from a BDT to a NN-based approach. Moreover, calibration of flavour tagging has bees introduced, as well as the use of flavour tagging techniques in boosted regimes.

\section{References}

[1] ATLAS Collaboration, The ATLAS Experiment at the CERN Large Hadron Collider, JINST 3 (2008) S08003.

[2] ATLAS Collaboration, Performance of the ATLAS track reconstruction algorithms in dense environments in LHC Run 2., Eur. Phys. J. C 77, 673 (2017).

[3] ATLAS Collaboration, ATLAS b-jet identification performance and efficiency measurement with $t \bar{t}$ events in pp collisions at $\sqrt{s}=13 \mathrm{TeV}$, Eur. Phys. J. C 79, 970 (2019).

[4] ATLAS Collaboration, Identification of jets containing b-hadrons with recurrent neural networks at the ATLAS experiment, ATL-PHYS-PUB-2017-003.

[5] ATLAS Colaboration, Deep Sets based Neural Networks for Impact Parameter Flavour Tagging in ATLAS, ATL-PHYS-PUB-2020-014.

[6] ATLAS Collaboration, Optimisation and performance studies of the ATLAS b-tagging algorithms for the 2017-18 LHC run, ATL-PHYS-PUB-2017-013.

[7] ATLAS Collaboration, ATLAS b-jet identification performance and efficiency measurement with $t \bar{t}$ events in pp collisions at $\sqrt{s}=13 \mathrm{TeV}$, Eur. Phys. J. C 79 (2019) 970.

[8] ATLAS Collaboration, Measurements of b-jet tagging efficiency with the ATLAS detector using $t \bar{t}$ events at $\sqrt{s}=13 \mathrm{TeV}$, JHEP 08 (2018) 89.

[9] ATLAS Collaboration, Expected performance of the 2019 ATLAS b-taggers, http://atlas.web.cern.ch/Atlas/GROUPS/PHYSICS/PLOTS/FTAG-2019-005/.

[10] ATLAS Collaboration, Variable radius, exclusive- $k_{T}$, and center-of-mass subjet reconstruction for Higgs $(\rightarrow b \bar{b})$ tagging in ATLAS, ATL-PHYS-PUB-2017-010.

[11] ATLAS Collaboration, Identification of Boosted Higgs Bosons Decaying Into b $\bar{b}$ with Neural Networks and Variable Radius Subjets in ATLAS, ATL-PHYS-PUB-2020-019. 